\title{
Cross-linguistic Spatio-temporal Association: Empirical Studies by Boroditsky
}

\author{
Yiqing Liao ${ }^{1}$, Rong Xiao, ${ }^{2, *}$ \\ ${ }^{1}$ School of Foreign Languages, Sun Yat-sen University, China \\ ${ }^{2}$ Department of English, the Chinese University of Hong Kong, China \\ *Corresponding Author: xiaorong19@126.com
}

Copyright $\mathrm{O} 2017$ by authors, all rights reserved. Authors agree that this article remains permanently open access under the terms of the Creative Commons Attribution License 4.0 International License

\begin{abstract}
This paper provides a critical review of cross-linguistic empirical research on spatio-temporal association among English and Chinese languages to see if it is possible to render the actual relations between language and reality in a systematic manner. It discusses a selection of relevant studies conducted by scholars within this domain. Hopefully this paper will shed light on the cross-linguistic studies of the relationship between language and thought in mental conceptualization. Implications are also given for future empirical research.
\end{abstract}

Keywords Spatio-temporal Association, Mental Conceptualization, Empirical Studies, Language and Thought

\section{Introduction}

While the strong version of linguistic determinism [1] has been controversial since its emergence, the question of "does language shape thought?" has never ceased to raise a number of linguists' attention.

Among all the subcategories of research in language and thought, one line that is of particular interest to many psycholinguists and cognitive linguists has been the influence of spatial expressions in a language on temporal perception by people using that language. The topic of spatial construal of time is actually not a novel one in studying the relationship between language and thought. In 1954, Piaget explored the connection between spatial and temporal reasoning in child development, however, it was Clark [2] who was the first to bring the issue of temporal concepts to the semantic grounding in language development. He proposes that children acquire expressions of space and time by learning how to apply these expressions to their prior knowledge about space and time. Following Clark and pushing his study further, a great many scholars of linguistics, psychology and cognition have worked on the spatial-temporal association of language and thought. For instance, Gentner [3] proposes and tests three possibilities to establish the conceptual role of space-time mappings: system-mapping, cognitive archaeology, and structural parallelism, finding that people's representation of time is actually structured in part by online structural analogies with the more concrete experiential domain of space. Vallesi, Binns \& Shallice [4] investigate how the abstract concept of time is represented in our cognitive system and suggest that one way in which the amount of elapsed time is cognitively represented is by the use of a spatial coordinate reference frame from left to right. And via investigating grammatically-prescribed prepositions, Kranjec et al. [5] demonstrate that the semantics of particular locative prepositions do constrain how we think about paired temporal concepts. Much evidence has pointed to the conclusion that people's perception of the abstract domain of time is derived from the more experience-based conceptualization of space: people tend to talk about time by employing spatial expressions.

However, the above mentioned studies are all mono-linguistically based and the existing research mostly takes English as the focus of study while leaving a great number of other languages unconsidered. This kind of language-specific studies has a fatal defect in the exploration of the general relationship between language and thought as they can only demonstrate the influence of a language on thinking in that particular language, the results of which cannot be safely generalized [6]. As Weist et al. [7] have noted, languages have diverse temporal and spatial coding systems which definitely cannot be inferred from any single language. Even the temporal metaphors vary across languages. Members of different language communities develop distinctive conceptual repertoires [8]. As a result, in recent years, the importance of cross-linguistic studies on spatio-temporal association has been increasingly recognized by scholars working in this field.

This paper critically reviews four empirical studies conducted by linguistic scholars on the spatial construal of temporal perception in Mandarin and English, as these four studies share similar objectives and adopt comparable 
research methodology. In fact each one of these studies is developed from the prior one(s), and could be taken as an extension or continuous refining of the previous studies. A critical analysis of these four empirical investigations and other relevant studies will hopefully shed light on the shortcomings of existing cross-linguistic studies on the relationship between language and thought and make implications for future empirical research.

\section{First Attempt: Language Shapes Thought}

Boroditsky's "Does language shape thought? Mandarin and English speakers' conceptions of time" [6] is among the first to examine the relationship between language and thought in relation to spatio-temporal associations in the cross-linguistic context and is the first to specifically address Mandarin and English as the testing languages. This work, although a controversial one - it is criticized by some scholars $[9,10]$ and later even by Boroditsky herself [11] is quite an influential study, as it opened a ground for empirical studies on cross-linguistic investigation of spatial and temporal mapping, partly because of this controversy.

In this study, three experiments are conducted concerning the spatio-temporal relationship in Mandarin and English to demonstrate that it is language and not other cultural factors that shapes people's habitual thought. A presupposition of this research is that while Mandarin speakers tend to talk about time vertically, native English users are more likely to adopt horizontal temporal expressions. Based on this premise, the author finds that this difference between the two languages has a strong influence on the ways their speakers think about time.

The participants of the first experiment are 26 native English speakers and 20 native Mandarin speakers at Stanford University. The participants are asked to answer questions about time after being exposed to spatial scenarios that are either horizontal or vertical and accompanied by a sentence description. The testing language is English and the participants are tested with English instructions. Questions are presented on a computer screen one at a time and participants' response times are measured and recorded by the computer. It is found that English speakers and Mandarin speakers tend to think differently about time. Native English speakers answer time questions faster after horizontal scenarios than after vertical scenarios, and they are also found to react faster for temporal questions phrased in horizontal terms than in vertical terms. While native Mandarin speakers' response times to temporal questions after horizontal scenarios and after vertical scenarios are almost the same. However, when considering the vertically phrased temporal questions, the native Mandarin speakers are found to react faster after vertical scenarios than after horizontal scenarios. This is contradictory with the native English speakers' results. In conclusion, Boroditsky proposes that people's experience with a language can shape the way they think.

Adopting a similar design and procedure, the second experiment examines the performance of 25 MandarinEnglish bilinguals to further test in what ways learning a new language influences people's thinking patterns. It is found that the propensity to think about time vertically is related to the length of pure Mandarin experience but not to the length of English experience. In the last experiment, 70 Stanford University undergraduates who are native English speakers are exposed to a set of 90 vertically phrased temporal questions to get used to this new way of talking about time and then they are asked to go through the procedures in Experiment 1. The results show that these participants tend to act more like native Mandarin speakers than untrained English speakers. As such, the author argues that learning a new way to talk about a familiar domain can change the way people think about that domain and that it is language alone that leads to the differences between English and Mandarin speakers' perception of time. Taking the findings of the three experiments together, Boroditsky then concludes that language indeed shapes people's habitual thought.

As mentioned above, Boroditsky's first attempt to demonstrate the relationship between language and thought through cross-linguistic empirical studies in Mandarin and English has received some criticism from other scholars. For instance, Chen, a Chinese scholar, replicated Boroditsky's research in Taiwan [9]. He finds that the presupposition in Boroditsky [6] that Mandarin speakers tend to talk about time vertically is untenable as Mandarin speakers actually tend to adopt horizontal spatial metaphors of time more frequently than vertical spatial metaphors. And when focusing on "month" as the time unit, Chen finds that Mandarin speakers show both trends of vertical and horizontal bias with equal chances which is contradictory to Boroditsky's findings. In conclusion, Chen claims that Boroditsky's study fails to prove that Mandarin and English speakers differ in their spatial conceptualizations of time and thus provides no evidence for linguistic relativity.

Chen's findings are further supported by January \& Kako [10] who conducted 6 replications of Boroditsky's research, all of which provide contradictory evidence to Boroditsky's original study. Besides, reviewing the third experiment in Boroditsky's research, January \& Kako find it untenable that 90 exposures to a new spatio-temporal metaphor can change native English speakers' perception of time. Thus, Boroditsky's [6] results cannot be considered as evidence for the Linguistic Relativity Hypothesis. Finally, the authors point out that while westerners may indeed typically order events in time horizontally from left to right when forced to do so [12], this does not necessarily reveal that their internal conceptualization of time is horizontally organized, and cultural convention may not be the single factor that drives this effect.

The two studies are highlighted here partly because of 
their insightfulness as critical empirical replications of Boroditsky's initial research, and partly because of their influence on Boroditsky's further attempts to examine the spatial conceptualization of time in Mandarin and English. Their doubts and critical remarks are clarified and rebutted in her later studies which will be discussed in the following sections.

However, besides these deficiencies pointed out by Chen [9] and January \& Kako [10], there are other things that need to be discussed critically in Boroditsky's initial attempt. Firstly, the participants in the three experiments should have been selected more carefully. All the subjects in the three experiments are English speakers - even the native Mandarin participants in Experiments 1 and 2 have at least 10 years of exposure to English. There is a fair reason to believe that the native Mandarin speakers' modes of thinking are at least to some extent influenced by the English language they have acquired - if it is really the case that language indeed can shape thought. Thus, these "native" Mandarin participants could not be taken as pure Mandarin speakers. To be more exact, they could only be considered as Mandarin speakers who have been influenced by the English language/thinking patterns or as Mandarin-English bilinguals. This indicates that Borodisky's research findings based on the so-called "native" Chinese speakers are not valid.

Secondly, the testing place should have been taken as a potential factor that might have influenced the results. The circumstantial elements might also have some effects on people's thinking modes. Staying in an English speaking context where all the communications are carried out in English, the participants might inevitably think according to the patterns characteristic of English [13]. This may partly explain why Chen [9] fails to replicate Boroditsky's findings in Taiwan.

And thirdly, all the three experiments were conducted in English with English instructions and English as the testing language describing the spatial scenarios and addressing the target questions. This was kept constant even when testing the "native" Mandarin speakers' spatial perception of time. In this case, it is not unreasonable to contend that the Mandarin speakers' temporal conception might have been affected by this exposure to English language, if the effect demonstrated in Experiment 3 is true that even a short-term exposure to a different spatio-temporal organisation can change people's perception of time. Moreover, people do not always speak their mind. Answering temporal questions or learning a new way of speaking does not necessarily mean that this process has actually occurred in people's mind. As January \& Kako [10] have noted, people sometimes are forced to speak in a certain way. To talk about time in horizontal/vertical terms in the experiments does not necessarily reveal that people's internal conceptualization of time is horizontally/vertically structured. Boroditsky's study only gives evidence for the fact that the Mandarin and English languages adopt different spatial-temporal representations, thus leading to different ways of talking about time - but not thinking about time. All these arguments imply that Boroditsky's initial research does not lend support to her conclusion that language shapes thought.

A lot of space has been devoted in this section to the discussion of this paper, because it lays the foundation for her further investigations on the relationship between language and thought in relation to spatio-temporal associations. The defects and shortcomings mentioned above are later taken into consideration and improved in Boroditsky's more recent works. The following three studies could be seen as a continuing refining process of Boroditsky's initial attempt.

\section{Second Attempt: Mandarin and English Speakers Think about Time Differently}

The second attempt at investigation of spatio-temporal mapping in Mandarin and English can be seen as a refinement of the above study and as a rebuttal of the criticism voiced by other scholars that it has only demonstrated that "Mandarin and English speakers talk about time differently" but not necessarily "have different lines of time conceptualization. The article "Do English and Mandarin speakers think about time differently?", published in 2010, was designed to specifically address the proposition that English and Mandarin speakers not only talk about but also think about time in different ways [11]

In this research, Boroditsky and her colleagues seem to be more cautious, and as a result of a more careful investigation, the original assumption of this research is refined to the statement that "vertical metaphors for time are more frequent in Mandarin than they are in English." This study could be taken as a reply to Chen's [9] findings that Mandarin speakers actually adopt horizontal expressions to talk about time more frequently than vertical terms, and it moves one step further by emphasizing the research domain as a comparison between English and Mandarin, and not in Mandarin itself.

Altogether, 181 participants in America take part in this study: 118 native English speakers and 63 Mandarin-English bilinguals. As we can see this choice of subjects demonstrates the same problem as Chen's [9] study: too few Mandarin speakers compared with the large number of native English speakers. However, in this new study, Boroditsky et al. take the participants' language proficiency into account, as is an improvement in comparison with the initial study. All the 118 native English speakers have no prior exposure to Mandarin. This is to ensure that their thinking patterns have not been influenced by another language. The 63 bilinguals are reported to be highly proficient in Mandarin. However, similar to Boroditsky [6], this experiment does not include any Mandarin 
monolinguals, so the comparison between Mandarin and English also seems untenable.

In the study, the participants were firstly asked to look at two pictures one after another in the same location on a computer screen, and then decided whether the second photo represented an earlier or later time by pressing one of two adjacent keys (either vertically or horizontally arranged) that were covered with black and white stickers which meant either 'earlier' or 'later' (with the left/upper key meaning "earlier" as the canonical situation and right/lower key "earlier" as the non-canonical situation). Thus, language was totally eliminated during the whole testing procedure.

Boroditsky et al. make a big step forward by employing nonlinguistic stimuli and responses. This replies well to January \& Kako's [10] claim that talking about time using spatial words does not entail that time is spatially conceptualized in people's mind. Nonlinguistic evidence helps to elucidate the relationship between space and time in the human mind. However, both the English speakers and the bilinguals have received English instructions before the test, which might suggest that this test is really English-oriented. In addition, the experiment is conducted in an English speaking environment, which might have had some influence on the Mandarin-English bilinguals' perception of time during their tests. The results of this study are presented in Figure1:

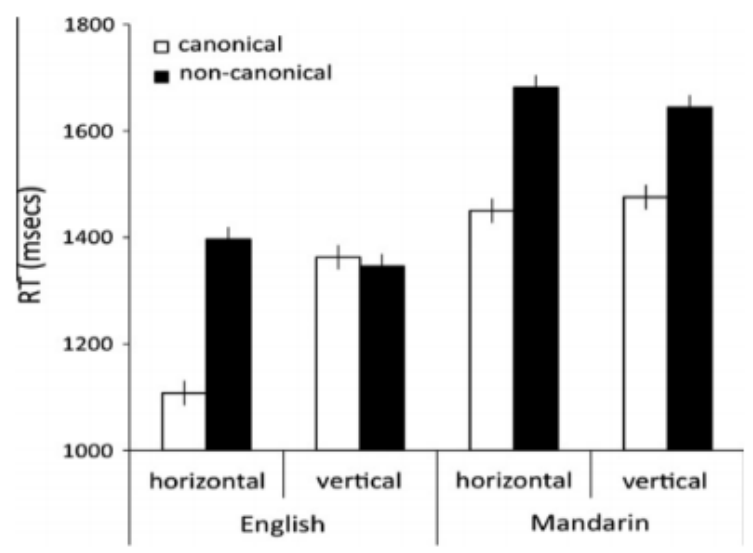

Figure 1. Native English and native Mandarin speakers' response times

The figure revealed that both English and Mandarin speakers demonstrate a canonicity effect on the horizontal axis, which indicates that speakers of both languages conceptualize time horizontally from left to right. However, in vertical condition, only Mandarin speakers (in this experiment the bilinguals) react faster when the "earlier" response key is on top than when it is in a lower position. The vertical canonicity does not have any effect on native English speakers - they even respond faster when the "earlier" key is at the bottom (which indicates a slight bottom-up direction in their time perception). Therefore, the authors conclude that while native English speakers tend to think about time horizontally from left to right, native
Mandarin speakers are equally good at both horizontal (from left to right) and vertical (from top to bottom) conceptualization of time. This is interpreted as evidence that Mandarin and English speakers think about time differently.

However, we have also noticed that the Responding Times (RT) for English speakers and Mandarin speakers are obviously different, with the English speakers responding (much) faster in both the horizontal and the vertical tests. This is a question worth thinking, yet left unexplained by the researchers - the authors have no interpretation for this finding. It might have been the case that the testing environment is biased in favor of the native English speakers as this experiment is conducted with English instructions and in an English speaking context.

Considering the above limitations of Boroditsky et al.'s 2010 study, much work still needs to be done in order to verify the proposition that Mandarin and English speakers indeed conceptualize time differently due to the different spatial-temporal expressions in their native languages.

\section{Third Attempt: Both Linguistic and Cultural Forces Shape Time Perception}

Fuhrman, McCormick, Chen, Jiang, Shu, Mao \& Boroditsky's paper entitled "How linguistic and cultural forces shape conceptions of time: English and Mandarin time in 3D" [14] continues to investigate how English and Mandarin speakers conceptualize time. As the title suggests, a major development of this study compared with the previous ones is that the researchers have stepped out of the circle of language per se, and have taken cultural elements into consideration when examining the factors influencing people's perception of time. This assumption contradicts Boroditsky's [6] claim that it is indeed language itself - and no other cultural factors - that shapes people's temporal conceptualization. And as we can see in the title, the authors of this study include both native English speakers and native Chinese speakers who have sufficient knowledge of their respective native languages and cultural backgrounds. This lays a firm foundation for a cross-linguistic study.

The experiments in this study take place in both America (for the English tests) and China (for the Mandarin tests), the native countries of the testing languages, thus minimizing the effects of language context on people's thought. Hence this study has an advantage over the previous ones by being carried out in a truly cross-linguistic context.

Moreover, the participants of this research are more carefully selected. The first experiment include 59 Stanford students who are native English speakers and whose English proficiency is claimed to be 5 based on a 1-5 scale, and none of them has had any exposure to Mandarin. The Mandarin speakers are also claimed to be proficient in 
Mandarin (5 out of 5) and none of them is reported a proficiency level in English of above 4 out of 5 . The English and Mandarin levels of EM bilinguals are also taken into consideration in Experiment 2. This language proficiency provides additional information about whether the participants could be taken as "pure" native speakers of a language or whether they are influenced by another language that might have exerted some influence on their ways of perception, thus adding weight to the objectivity and credibility of the experiments.

Like the second attempt, this study also uses non-linguistic stimuli, but unlike the previous investigation, the instructions are presented in the participants' native languages, thus reducing the short-term influence of non-native languages on native speakers' perception of time. Besides, this research also expands from a two-dimensional (horizontal-vertical) space examined in the two previous studies to a three-dimensional (transverse-vertical-sagittal) space, an improvement which is already mentioned in the second study, but not applied until this investigation. The results of the first experiment in this study are presented below:

Table 1. English and Mandarin speakers' average response times (ms)

\begin{tabular}{lcc}
\hline & English & Mandarin \\
\hline Left is earlier & $* 936 *$ & 1675 \\
Right is earlier & 1045 & 1793 \\
Near is earlier & 1015 & 1745 \\
Far is earlier & 983 & 1666 \\
Top is earlier & 974 & $* 1609 *$ \\
Bottom is earlier & 993 & 1853 \\
\hline
\end{tabular}

This table shows the average response times of the English and Mandarin speakers along the three dimensions (both canonical and non-canonical). It shows that native English speakers give the fastest responses when 'left' represents 'earlier', and the Mandarin speakers when 'top' stands for 'earlier'. This demonstrates that Mandarin speakers tend to think about time vertically (with the direction from top to bottom) rather than transversely. This finding actually is in contrast with the results of Boroditsky et al.'s [11] second study presented in Figure 1, which indicates that Mandarin speakers have a tendency to think about time horizontally more than vertically just as the English speakers do. Besides, Table 1 reveals that English speakers also show a slight tendency to think about time vertically as they respond a bit faster when 'top represents earlier' than when 'bottom represents earlier'. This is also contradictory to Boroditsky et al.'s [11] results. We can reasonably attribute these contradictions to the different language proficiencies of the participants and the different experiment environments in the two investigations. Moreover, just as in the previous study, the English speakers in the present study also react much faster than the Mandarin speakers. The explanation of the observation that the researchers offer this time is that the English speakers are more familiar with participating in psychological experiments.

The second experiment in this study is designed to explore the factors influencing people's internal conceptualization of time. The findings are presented in Figures 2 and 3:
- vertical

\section{horizontal}

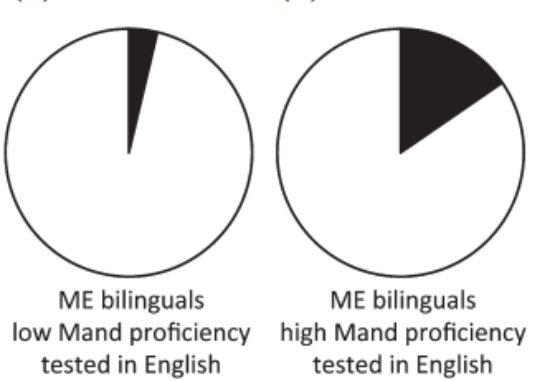

(D)

(E)

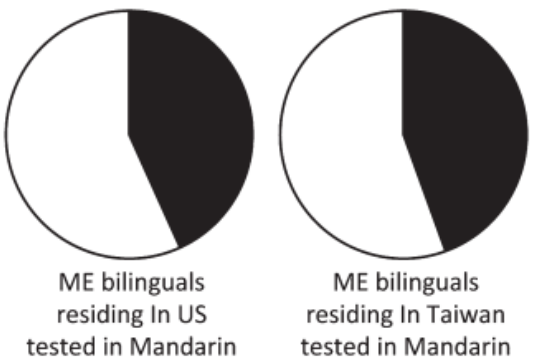

Figure 2. Different participant groups' tendency for time perception. Part 1 
(A)

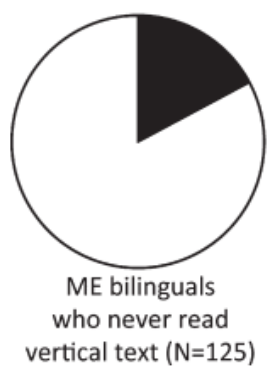

(B)

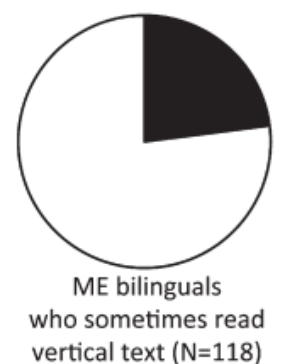

- vertical horizontal

Figure 3. Different participant groups' tendency for time perception. part 2

As shown in Figures 2 and 3, this study further proposes that English and Mandarin speakers' perceptions of time are influenced by five factors: proficiencies in Mandarin and in English, the language of the test, the country of the test and experience with a vertical text, instead of by language alone as claimed in Boroditsky's [6] first study.

Based on the findings presented in Figure 2, the authors further claim that short-term language exposure plays a significant role in people's perception of time. However, the question remaining unanswered is whether this difference really occurs due to the different testing languages? When taking a closer look at the participants in these groups, we find that this claim cannot be justified. In their paper, the authors report that the bilingual participants in groups D and $\mathrm{E}$ are native Mandarin speakers and thus are tested in Mandarin. And for group $\mathrm{C}$, the authors do not mention whether the participants are native Mandarin speakers or not - and it tends to be not so according to the arrangement of this experiment (otherwise the comparison between the first three groups would be untenable). Thus, it is quite reasonable to assume that the different levels of vertical tendency of different groups may not have been the result of the short-term influence of the testing languages, instead they are more likely to result from the participants' long-exposure to the language they are tested in. Besides, the number of bilingual participants tested in English (170) is much larger than the group of native Mandarin bilingual participants tested in Mandarin (47). Given this questionable identity of the participants in group $\mathrm{C}$ and the biased distribution of participants among different groups, it is clear that this study fails to demonstrate the immediate influence of language on people's temporal conception.

Furthermore, although the participants in this research differ in their Mandarin proficiency levels from non to highly proficient, their English language levels are from good to quite good (with the group lowest level equal to 3.53). It is hard to justify that the highly competent Mandarin speakers' inner conceptualization of time has not been influenced by their good to very good English proficiencies.

Thus far, we can see that although the third attempt has improved the research methodology compared with the previous studies, in particular in the selection of participants, and it has expanded the scope of the previous studies, it is still insufficient to be taken as an evidence for different spatio-temporal mapping patterns in Mandarin and English. Further empirical studies remain to be done.

\section{Fourth Attempt: Immediate and Chronic Influence of Spatio-temporal Metaphors on Time Perception}

This is one of the latest researches investigating the space-time relationship in English and Mandarin and is a continuation of the previous attempt [11] to demonstrate that both immediate and chronic factors exert influence on people's perception of time. Two experiments are conducted in this research to investigate the influence of spatio-temporal metaphors in a language on its speakers' conceptualization of time [13].

The subjects of this research were even more carefully selected than in any of the previous studies. They included both Mandarin/English monolinguals and bilinguals. In order to minimize possible adverse effects of any other languages on the subjects, the group of Mandarin monolinguals comprised only those whose English level was around 1 (out of 5), and who did not know Cantonese. Likewise, all the English monolinguals had no prior exposure to Chinese. The English monolinguals and the ME bilinguals were tested in America and the Mandarin monolinguals were tested in Taiwan.

The first study was divided into two questions. For the first question, the native English group and the ME-bilingual group were tested in English, and the native Mandarin group was tested in Mandarin. The results from the two groups tested in English showed that unlike the English monolinguals who took an ego-moving perspective in temporal conceptualization, the $\mathrm{ME}$ bilinguals demonstrated a tendency to adopt a time-moving perspective which was in line with the Mandarin monolinguals. Then the same groups were tested for another question. This time, both the Mandarin monolinguals and ME bilinguals were tested in Mandarin, while the native English group was tested in English. A comparison between the first two groups revealed that the ME bilingual group conceptualized time from an ego-moving perspective which was similar to the English monolinguals' perception of time. In this study, the possibility of short-term influence of the testing language (by only comparing the groups tested in the same language) and testing location (as the bilinguals were only tested in America) is also considered and controlled.

The results from this study demonstrate firmly that bilinguals import temporal conceptual structures both from L1 (Chinese) to their perception of temporal metaphors in L2 (English) (as in the first question) and from L2 to L1 (as in the second question). This is an extension and fine-tuning of the more general conclusion that the spatio-temporal 
metaphors in a language shape their speakers' internal conceptualization of time, which is an unceasing topic in Boroditsky and her colleagues' previous researches.

However, as Lai and Boroditsky pointed out themselves, the same bilingual group was tested for two different questions in this study. It would be interesting to test this group on one and the same question in both Mandarin and English to see whether on-the-spot linguistic exposure could affect their perception of time. But again, in such a study, the bilinguals' native language and the testing locations all need to be taken into consideration to ensure that the differences in the participants' temporal perception is indeed caused by the testing languages and not by their long-term exposure to a language and the general language environment of the test.

The second experiment of this study was specifically designed to test whether short-term exposure to different spatio-temporal metaphors can affect people's conceptualization of time. In this experiment, the $\mathrm{ME}$ bilinguals were tested both in America and Taiwan in Mandarin. The subjects were asked (front-back or up-down) temporal questions, and had to point to the space around them to locate these time points (3-D: up-down, left-right, front-back). The results showed that the subjects arranged time vertically when prompted with up-down metaphors and sagittally when prompted with front-back metaphors in Mandarin. The authors claimed that Mandarin speakers were more likely to conceptualize time along the front-back axis when understanding front-back metaphors and more likely to construct up-down representations of time when understanding up-down metaphors. Based on this finding, the authors further concluded that different spatio-temporal metaphors have in-the-moment influences on people's conceptualization of time.

But this could hardly be taken as evidence for immediate influence of spatio-temporal metaphors on time perception.

Firstly, the results of this experiment seem to be self-evident: there would be no reason for language users to conceptualize time vertically when processing horizontal spatio-temporal metaphors. It is both a waste of energy and abnormal way of thinking and communicating according to Cooperative Principle [15] and Relevance Theory [16] in conversational analysis. In other words, the findings of this study are universally applicable: the same might be true in other languages and cultures. The results of this study could only be taken as evidence for the fact that spatio-temporal metaphors are still psychologically alive in Mandarin and exist in line with people's perception of time and space, but provide no evidence for the linguistic relativity hypothesis.

Besides, a closer look at the sample questions reveals that the subjects' responses might have been premised by the questions, and did not necessarily entail the participants' thinking in that way. For example,

* “假设这里是这个礼拜, 你认为前一个礼拜在哪里? 后一个礼拜在哪里?"

* "suppose this here is this classifier-ge week, you think front one classifier-ge week locate where? Back one classifier week locate where?"

* translation: "suppose here is this week, where do you think is last week? where do you think is next week?" (adapted from the original study)

As thus, the concepts of "front" and "back" were used explicitly in the prompting questions, thus, it was easy for the subjects to adopt these concepts while responding to the questions, but this does not necessarily mean that the subjects thought about time in that way. Besides, it is worth noticing here that for expressing "last week" and "next week”, excepts “前一个礼拜 (front one classifier-ge week)” and “后一个礼拜(back one classifier week)”, in Mandarin, people also say “上一个礼拜 (upper one classifier-ge week)” and “下一个礼拜 (lower one classifier-ge week)”. Therefore, it might be possible that the responses in this experiment did not truly reflect people's inner perception of time, but were simply conditioned responses to what they had heard and remembered a few seconds ago.

Thirdly, even if the results are indeed reflections of bilinguals' perception of time, is this perception really caused by the immediate influence of the questions posed by the experimenters? This is another question which needs to be explored. As the authors claim, the prompting questions used in this study were "conventional metaphoric expressions in Mandarin, not novel constructions", which means that the current study adopted a conventional way of expressing spatio-temporal metaphors in Mandarin. As the participants were highly proficient in Mandarin (4.48 and 5 out of 5), and for those who were tested in Taiwan, they were native mandarin speakers, it is reasonable to assume that all these subjects had been indeed affected by the Mandarin way of processing spatio-temporal metaphors for a long time and to a great extent. The questions delivered to them were in line with their habitual ways of talking about time, thus, the effect of the questions on the participants' perception of time should rather be interpreted as a long term influence than on-the-spot influence of this experiment.

Moreover, the authors noticed that subjects who were tested in America were more likely to use the left-right axis than those in Taiwan and less likely to use the front-back axis. They interpreted this finding as the result of different testing locations. The testing locations of course, might be an influencing factor, but another factor is still missed here - the participants' English proficiency. As we can see from the original study, although both groups had high proficiency in Mandarin, the bilinguals in America had a mean English proficiency of 4.01, and the bilinguals in Taiwan only 2.71 . As it has been proven that proficiency levels of a language may influence people's perception of time in another language [14], due weight should be given to the different English levels of the participants when investigating and interpreting the participants' different patterns of temporal perception. 


\section{Conclusion and Implications for Further Research}

The discussion presented in the paper illustrates a continuing perfecting of empirical studies on the temporal-spatial association in Mandarin and English to support the linguistic relativity hypothesis. It also reflects the kinds of problem that many researchers face when doing cross-linguistic and cross-cultural research in language and cognition.

Researchers have made a great progress from the initial attempt to testify that language shapes thought in general and then restricted it to the more specific conclusion about Mandarin and English. Later this continuous attempt was extended to include both language and culture as the influencing factors on Mandarin and English speakers' different modes of temporal perception. Then more recently, the linguistic influence on people's conceptualization of time was examined from both immediate and chronic perspectives. As the research goes thoroughly on, the author(s) tends to be more cautious in the selection of participants, testing materials and the design of testing procedures. This also reflects the general tendency in the study of the relationship between language and thought in relation to spatio-temporal mappings.

However, there are still defects in the existing literature, as are exemplified in the last two attempts: although the English and Mandarin proficiency levels of the participants were considered and restricted, the selection was still based on the subjects' self-reported English and Mandarin levels. The participants' language proficiency was not tested in an objective way. It is sometimes hard to distinguish one level from another subjectively even for a monolingual user, not to mention there are so many bilingual participants (in one research around 200). And the subjects' self-perception of their language levels, just as their temporal perception, might have been affected by other internal or external factors, such as their personalities - some people just tend to be more cautious or modest while making estimations.

In the meantime, the proposition that language has an immediate effect on temporal perception needs further exemplification. Existing experiments are either designed as testing different groups of participants on the same token (as in the second experiment of the third attempt) or the same group tested in different tokens (as in the first experiment of the fourth attempt). The experiment specially designed to test in-the-moment effect of spatio-temporal metaphors on people's perception of time (the second experiment in the fourth study) falls into the infinite loop of "people tend to think in A when talking about A". Actually, this loop is untenable unless alternative explanatory pathways (e.g., there would be possibility for people to "think in B when talking about A") can be ruled out. There is still no sufficient evidence that immediate situational linguistic factors shape people's perception of time.

Moreover, the existing research has demonstrated firmly that Mandarin and English speakers have different temporal conceptualization models, and why this occurs: due to different spatio-temporal expressions in the two languages and other cultural factors such as the writing and reading directions. One domain which needs further exploration is concerned with how the different time-perception models in the two languages occur. As it was already exemplified, English and Mandarin have different temporal perceptions because of their different spatio-temporal metaphors, but in what ways the English/Mandarin metaphors are mapped onto their respective speakers' perceptions of time is still an unexplored area. Cross-linguistic studies have found time and again that some, but not all, spatial lexemes can be used in temporal language, and different languages have different conflations of temporal cognition and spatial expression, what calls for further exploration is the situation in Mandarin and English.

Besides, cross-linguistic studies which focus on the spatial and temporal systems in children have found that temporal and spatial systems emerge simultaneously and have their respective statuses in children's mind, thus rejecting the claim that temporal distinctions are derived from spatial differences [7]. And in a more recent study on a Turkish sign language, Arik [17] notices mismatches between the participants' temporal expression and the deictic use of the front-back axis, suggesting that temporal and spatial language may not be derived from each other but might only share some properties at the lexical level. Thus, further empirical studies with convictive designs remain to be conducted to support Sapir-Whorf linguistic relativity hypothesis through the spatio-temporal mapping of language and cognition.

\section{REFERENCES}

[1] Whorf, B. L. In: J. B. Caroll (ed.): Language, thought and reality: Essays by B. L. Whorf. MIT Press: Cambridge, MA 1956.

[2] Clark, H. H.: "Space, time, semantics, and the child". In: T. E. Moore (ed.): Cognitive development and the acquisition of language. Academic Press: New York 1973.

[3] Gentner, D.: "Spatial metaphors in temporal reasoning". In: M. Gattis (ed.): Spatial schemas in abstract thought. MIT Press: Cambridge, MA 2001, pp. 203-222.

[4] Vallesi, A., Binns, M. A., Shallice, T.: "An effect of spatial-temporal association of response codes: understanding the cognitive representations of time". Cognition. 107(2), 2008, pp. 501-527.

[5] Kranjec, A., Cardillo, E. R., Schmidt, G. L. \& Chatterjee, A.: "Prescribed spatial prepositions influence how we think about time". Cognition, 114, 2010, pp. 111-116.

[6] Boroditsky, L.: "Does language shape thought? Mandarin and English speakers' conceptions of time". Cognitive Psychology, 43(1), 2001, pp. 1-22. 
[7] Weist, R. M., Atanassova, M., Wysocka, H. \& Pawlak, A.: "Spatial and temporal systems in child language and thought: A cross-linguistic study". First Language, 19(57), 1999, pp. 267-308.

[8] Casasanto, D. \& Boroditsky, L.: "Time in the mind: Using space to think about time". Cognition, 106, 2008, pp. 579-593.

[9] Chen, J. Y.: "Do Chinese and English speakers think about time differently? Failure of replicating Boroditsky (2001)". Cognition, 104(2), 2007, pp. 427-436.

[10] January, D. Y. \& Kako, E.: "Re-evaluating evidence for linguistic relativity: Reply to Boroditsky (2001)". Cognition, 104(2), 2007, pp. 417-426.

[11] Boroditsky, L., Fuhrman, O., \& McCormick, K.: "Do English and Mandarin speakers think about time differently?" Cognition, 118(1), 2010, pp. 123-129.

[12] Tversky, B., Kugelmass, S. \& Winter, A.: "Cross-cultural and developmental trends in graphic productions". Cognitive Psychology, 23, 1991, pp. 515-557.
[13] Lai, V.T. \& Boroditsky, L.: "The immediate and chronic influence of spatio-temporal metaphors on the mental representations of time in English, Mandarin, and Mandarin-English speakers". Frontiers in Psychology, 4, 2013, pp. 142-164.

[14] Fuhrman, O., McCormick, K., Chen, E., Jiang, H., Shu, D. F., Mao, S.M. \& Boroditsky, L.: "How linguistic and cultural forces shape conceptions of time: English and Mandarin time in 3D”. Cognitive Science, 35, 2011, pp. 1305-1328.

[15] Grice, H. P.: "Logic and Conversation". In: C. Peter \& M. Jerry (eds.): Syntax and Semantics (Vol.3). Academic Press: New York 1975.

[16] Sperber, D. \& Wilson, D.: Relevance: Communication and Cognition. Harvard University Press: Cambridge, MA 1986.

[17] Arik, E.: "Space, time, and iconicity in Turkish sign language (TID)". Trames, 16(4), 2012, pp. 345-358. 